\title{
Endoscopic balloon dilatation of duodenal strictures in Crohn's disease
}

\author{
SM Kelly, JO Hunter
}

\begin{abstract}
Summary
Endoscopic balloon dilatation of oesophageal and colonic strictures is now widely performed. Duodenal strictures due to Crohn's disease represent a difficult clinical problem as surgical intervention is a major undertaking and recurrences are common. We describe endoscopic balloon dilatation in three patients with obstructive symptoms from duodenal strictures due to Crohn's disease. The procedure appeared safe, was well tolerated and resulted in an excellent symptomatic response. This method is particularly suitable for short duodenal strictures and avoids the need for surgical intervention in this difficult group of patients.
\end{abstract}

Keywords: duodenal strictures, Crohn's disease, endoscopic dilatation

Crohn's disease involves the duodenum in approximately $1-2 \%$ of cases. ${ }^{1}$ Mucosal oedema, thickening, ulceration, and nodularity are typical features and, as with more distal Crohn's disease, progressive fibrosis and stricturing is common. Upper abdominal pain, nausea, vomiting and weight loss are the usual symptoms. There are a number of management options in such cases (box 1). Surgical intervention is common and in one series ${ }^{2} 37 \%$ of patients with duodenal Crohn's required surgical intervention, in the form of either a resection or a by-pass procedure. Unfortunately, as with Crohn's disease elsewhere in the gastrointestinal tract, recurrence is common. Recognition that recurrence is common, and that resection is often not curative, has led to a conservative surgical approach in these patients. This includes the performance of limited resections and stricturoplasty where possible. ${ }^{3}$ The main indication for surgery in Crohn's disease is that of fibrous strictures resulting in obstruction. Strictures elsewhere

Department of

Gastroenterology,

Addenbrooke's

Hospital, Cambridge,

UK

SM Kelly

JO Hunter

Correspondence to

Dr JO Hunter, Unit E7,

Addenbrooke's Hospital,

Cambridge CB2 2QQ, UK in the gastrointestinal tract can be treated endoscopically with balloon dilatation. ${ }^{4}$ Oesophageal strictures are routinely managed with endoscopic balloon dilatation and some cases of pyloric obstruction are also amenable to dilatation, avoiding the need for surgical intervention. ${ }^{5}$ Recent reports have demonstrated that ileocolonic anastomotic and other colonic strictures can be managed by dilatation using 'through the 'scope' (TTS) balloons (Regiflex Pyloric TTS, Boston Scientific Corporation, St Albans, UK). ${ }^{6}$ These balloons can be passed through the biopsy channel of the endoscope and positioned accurately across the stricture under direct vision before inflation. In this paper we report the successful management of three patients with Crohn's duodenal strictures by endoscopic balloon dilatation, thereby avoiding surgical intervention.

\section{Patients and methods}

Endoscopic balloon dilatation was performed on three patients attending the Department of Gastroenterology, Addenbrooke's Hospital, Cambridge. These patients all had radiological and histologically proven Crohn's disease.

\section{Patient 1}

A 39-year-old woman with a six-year history of ileocolonic Crohn's disease presented with a five-month history of weight loss and upper abdominal bloating with vomiting. A barium meal revealed a massively dilated stomach with a short tight stricture at the junction of the first and second parts of the duodenum (figure (A)).

\section{Patient 2}

A 51-year-old woman with small bowel Crohn's presented with weight loss and upper abdominal fullness. A barium study demonstrated a dilated stomach and first part of the duodenum with a $2-\mathrm{cm}$ stricture in the second part.

\section{Patient 3}

A 30-year-old woman with a seven-year history of ileocolonic Crohn's disease presented with upper abdominal fullness, pain and weight loss. A barium study revealed a pinhole stricture at the junction of the first and second parts of the duodenum.

All three patients underwent endoscopy with balloon dilatation of these strictures. After 


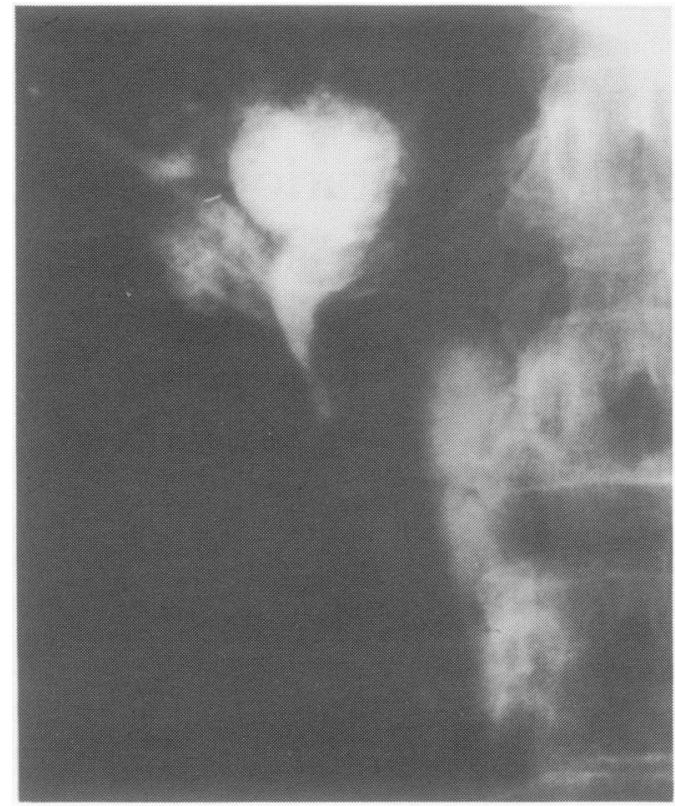

A

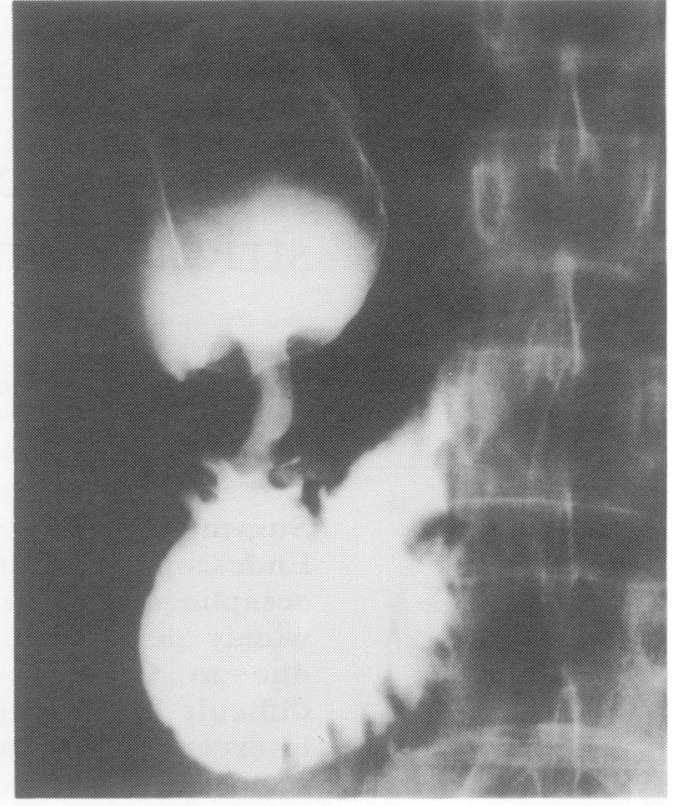

B

Figure (A) Barium study demonstrating a tight stricture in the duodenum with very little passage of contrast. (B) Repeat examination following endoscopic balloon dilatation, revealing widening of the stricture and passage of contrast into the distal duodenum. This correlated with symptomatic improvement

Ltd, Tring, UK) and pethidine, patients underwent examination with a standard gastroscope (Q20, Olympus-Keymed, Southend, UK). The stricture was identified and an 8-mm TTS balloon passed across the stricture under direct vision. The balloon was then inflated to 60 psi for one minute, followed by deflation and re-inflation for a further minute. Radiological screening was not required. Patients were observed overnight as in-patients. The procedure was well tolerated and there were no complications.

\section{Results}

All three patients had an excellent symptomatic response. They were able to resume a normal diet and experienced no pain or bloating. All three gained weight and repeat barium examinations revealed improvement in the strictures with free passage of barium (figure (B)). Patient 1 had a recurrence of her symptoms after 12 months and underwent a successful repeat dilatation which was performed as an outpatient. At a mean follow-up period of 19 months all three patients are well and asymptomatic from their duodenal Crohn's disease.

\section{Discussion}

Duodenal Crohn's disease is uncommon but when duodenal strictures occur management is difficult. Upper abdominal pain, vomiting, and

1 Fransden PJ, Jarnum S, Malmstrom J. Crohn's disease of the duodenum. Scand 7 Gastroenterol 1980; 15: 683-8.

2 Nugent FW, Roy MA. Duodenal Crohn's disease: an analysis of 89 cases. Am $\mathcal{f}$ Gastroenterol 1989; 84: 249-54.

Dehn TCB, Kettlewell MGW, Mortensen NJ, Lee ECG, Jewell DP. Ten year experience of stricturoplasty for obstructive Crohn's disease. Br F Surg 1989; 76: 339-41.

4 Linder KD, Ott BJ, Hughes RW. Balloon dilatation of upper digestive tract strictures. Gastroenterology 1985; 89: 339-41.

\section{Learning points}

- Crohn's disease involves the duodenum in $1-2 \%$ of cases

- surgical intervention for duodenal obstruction is often unsatisfactory

- endoscopic balloon dilatation of such

strictures is now possible with good results

Box 2

weight loss are common symptoms. Surgical intervention, in the form of either a resection or a by-pass of the stricture, is a major procedure and results in further surgery in up to $25 \%$ of cases. ${ }^{2}$ However, this paper demonstrates that duodenal strictures resulting from Crohn's disease can be successfully managed by endoscopic balloon dilatation. The only technical problem encountered was that in one patient the stomach was so dilated, as a result of the duodenal obstruction, that the endoscope could only just reach the stricture itself. Otherwise the dilatations were relatively straightforward With the advent of technical improvements and wider availability of small bowel enteroscopy, it may be possible to treat more distal Crohn's strictures with endoscopic balloon dilatations in the future.

This treatment is suitable for short strictures. It appears safe and well tolerated and can overcome the need for surgical intervention in this difficult group of patients.

5 Hogstrom M, Haglund U. A technique for endoscopic Hogstrom M, Haglund U. A technique for endoscopic balloon dilatation of pyloric stenoses. Endoscopy 1985; 17

6 Williams AJK, Palmer KR. Endoscopic balloon dilatation as a therapeutic option in the management of intestinal stric tures resulting from Crohn's disease. Br $₹$ Surg 1991; 78: 453-4. 